Article

\title{
Time-Dependent Production of the Bioactive Peptides Endolides A and B and the Polyketide Mariline A from the Sponge-Derived Fungus Stachylidium bicolor 293K04
}

\author{
Celso Almeida ${ }^{1,2, *}$, Gerald Bills ${ }^{1,3}$, Víctor González-Menéndez ${ }^{1}$, Jesús Martin ${ }^{1}$, \\ José R. Tormo ${ }^{1}$ (i) and Olga Genilloud ${ }^{1}$ \\ 1 Fundación MEDINA, Avenida del Conocimiento 34, Parque Tecnológico de Ciencias de la Salud, \\ E-18016 Granada, Spain; billsge@vt.edu (G.B.); victor.gonzalez@medinaandalucia.es (V.G.-M.); \\ jesus.martin@medinaandalucia.es (J.M.); jose.tormo@medinaandalucia.es (J.R.T.); \\ olga.genilloud@medinaandalucia.es (O.G.) \\ 2 UCIBIO-REQUIMTE, Departamento de Ciências da Vida, Faculdade de Ciências e Tecnologia, \\ Universidade Nova de Lisboa, 2829-516 Caparica, Portugal \\ 3 Texas Therapeutics Institute, The Brown Foundation Institute of Molecular Medicine, The University of \\ Texas Health Science Center at Houston, 1881 East Road, Houston, TX 77054, USA \\ * Correspondence: celsoguerreiro@gmail.com, Tel.: +34-958-993-965; Fax: +34-958-846-710
}

Received: 11 August 2017; Accepted: 1 September 2017; Published: 5 September 2017

\begin{abstract}
Previous investigations of the sponge-derived fungus Stachylidium bicolor (S. bicolor) 293K04 led to the isolation of the biosynthetically unusual polyketides marilines A-C and the bioactive tetrapeptides endolides A-B, identified as potential neuropathic drug leads. Furthermore, prior extended solid cultivation of S. bicolor $293 \mathrm{~K} 04$ for 60 days resulted in a significant increase of polyketide yield, and the isolation of seven new polyketides. Due to the interest in endolide activity, unusual biosynthetic diversity, and the late stage polyketide production, we studied the cultivation conditions for determining the production time distribution and yields of these secondary metabolites. Results indicated a first production phase of secondary metabolite dominated by peptides, after 21-23 days. Polyketide mariline A1/A2 only started at day 35 of growth, an unusually late period for secondary metabolite expression. This unusual bimodal sequential expression of different families of secondary metabolites suggests value in exploring extended cultivation times to identify novel bioactive fungal compounds.
\end{abstract}

Keywords: fungi; natural products; fermentation; production conditions

\section{Introduction}

Filamentous fungi are prolific producers of a broad array of low-molecular-weight secondary metabolites affecting a wide range of cellular processes including cell signaling, development, and intercellular communication [1]. In addition, a select group of these compounds have been developed in the clinic with important applications such as antibiotics, immunosuppressors, and other chemotherapeutic agents. Microbial genome mining efforts to explore fungal biosynthetic potential have shown that the fungal secondary metabolome has been substantially underestimated and that a large majority of fungal secondary metabolite biosynthetic gene clusters may remain silent under laboratory cultivation conditions [1,2].

Previous research exploring the chemistry of the strain Stachylidium bicolor 293K04 led to the isolation of more than 15 new secondary metabolites, including the recently reported receptor-binding $5 \mathrm{HT} 2 \mathrm{~b}$ and vasopressin $1 \mathrm{~A}$ bioactive peptides endolides A and B [3] (Figure 1), which have shown 
potential as neuropathic drug leads with potent in vivo bioactivity (Almeida, C., University of Bonn, Bonn, Germany, unpublished work, 2017). The low yield obtained from culture extracts of S. bicolor 293K04 currently hinders further in vivo pre-clinical development. Interestingly, endolides incorporate a 3-(3-furyl)-alanine residue, a rare amino acid previously reported only twice. The first description corresponds to rhizonin A, a peptide described as produced by the zygomycete fungus Rhizopus microsporus [4,5], and later confirmed to be encoded by the symbiotic endobacterium Burkholderia endofungorum [6]. The second report of 3-(3-furyl)-alanine was associated with the cytotoxic bingchamides from Streptomyces bingchenggensis [7], reinforcing the hypothesis of a possible bacterial biosynthetic origin of this rare amino acid. The sponge-derived fungus S. bicolor 293K04 also produces the unprecedented bioactive polyketide-like phthalimidine and phthalide derivatives, marilines (Figure 1), marilones, maristachones, cyclomarinone and marilactone. Their biosynthesis was suggested to require unique and/or rare reactions in fungal secondary metabolism, such as the methylation of the acetate starter unit of the phthalidmidine/phthalide core structure, or the hydroxycyclopentenone ring biosynthesis of cyclomarinone, predicted to be a new carbon arrangement among polyketides [8-10]. These polyketides exhibited potent bioactivity in several bioassays, with mariline A1/A2 enantiomers exhibiting human leucocyte elastase inhibition (HLE) with an IC50 of $0.86 \mu \mathrm{M}$, as well as antiproliferative activity against cancer cells, antiplasmodial activity, and antagonistic activity against several psychoactive receptors [9].

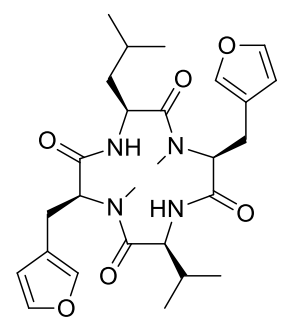

(1)

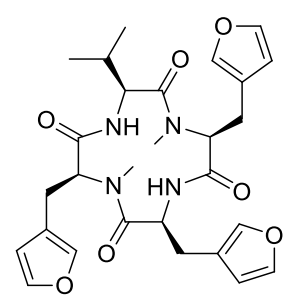

(2)

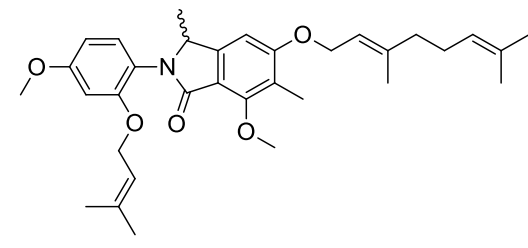

(3)

Figure 1. Structure formulae of endolides A and B (1 and 2 respectively) [3] and Mariline A1/A2 (3) [9].

Besides polyketides, chemical analysis of extracts of S. bicolor 293K04 led to the isolation of the tyrosine derivatives stachylines A-D [11] which incorporate a rare terminal oxime group that occurs as an interchangeable mixture of $\mathrm{E} / \mathrm{Z}$ - isomers.

The endolides yields from solid cultures of S. bicolor (ca. $6 \mathrm{mg} / \mathrm{L}$ ) are insufficient for in vivo pre-clinical development. Thus, optimized liquid scaled-up fungal fermentations are needed to progress the lead compound. Furthermore, a 40-day fungal cultivation using 10-L solid media in Fernbach flask format permitted the isolation of a large amount of peptides endolide $\mathrm{A}$ and B [3] and a minimal amount of the polyketides marilines A1/A2, marilones A and C $[9,10]$. Interestingly, an experimental 60-day $10 \mathrm{~L}$ solid cultivation of the fungus yielded low amounts of the peptidic endolides, but increased amounts of polyketides mariline A1/A2 (3-fold) or marilone A (8-fold), and seven new polyketides found exclusively in the extracts of the 60-days cultivations [8-10] which we reason was an unusually delayed stage of polyketide production.

Most secondary metabolites in liquid cultivations are generally considered to initiate production with the transition to the stationary phase. For the purpose of convenience and rapid production schedules, fungal cultivations for secondary metabolites usually are not grown for extended times. The biphasic production of unprecedented and complex marilines and marilones suggested that solvent extractions of late-stage cultivations could be a simple method for the identification of novel bioactive secondary metabolites. Production of such late-phase metabolites could be triggered by natural shifts resulting from the late stage cultivation conditions, the influence of toxic byproducts, and nutrient limitation [12,13]. 
The purpose of this study has been to characterize liquid format cultivations and the effect of fermentation parameters and media composition on the temporal production and the yields of secondary metabolites isolated from S. bicolor 293K04.2.

\section{Materials and Methods}

\subsection{Fungal Material}

The marine-derived fungus Stachylidium bicolor 293K04 was provided by G. Koenig, Institute for Pharmaceutical Biology, University of Bonn, Bonn, Germany. The fungus was isolated from the sponge Callyspongia cf. flammea, which was collected at Bare Island, Botany Bay, New South Wales, Australia, in 1997. It was identified by P. Massart and C. Decock, CCM/MUCL, Catholic University of Louvain, Louvain-la-Neuve, Belgium. Phylogenetic analysis by ITS/28S rDNA sequences determined the species as S. bicolor (Almeida, C., Fundación MEDINA, Granada, Spain, unpublished work, 2017). A specimen was deposited at the Institute for Pharmaceutical Biology, University of Bonn (Bonn, Germany), isolation number "293K04", running number "220", and is available upon request.

\subsection{General Experimental Procedures}

\subsubsection{Cultivation Procedures}

Fifty-mL cultures (in $250 \mathrm{~mL}$ Erlenmeyer flasks) were inoculated with $1.5 \mathrm{~mL}$ ( $3 \% v / v$ final culture volume) of a seed culture. The seed culture in SMYA medium (maltose $40 \mathrm{~g}$, yeast extract $10 \mathrm{~g}$, neopeptone $10 \mathrm{~g}$, agar $4 \mathrm{~g}$ per $\mathrm{L}$ of deionized $\mathrm{H}_{2} \mathrm{O}$ ) was inoculated with 5-10 agar plugs per $50 \mathrm{~mL}$ of SMYA and fermented for about $7 \mathrm{~d}$. EPA (Environmental Protection Agency) vial cultivations $(10 \mathrm{~mL})$ were inoculated with $0.3 \mathrm{~mL}(3 \% v / v$ final culture volume $)$ of seed culture in SMYA. Biomalt with added artificial sea water-BMS—contained Villa Natura Kraftnahr biomalt ${ }^{\circledR}$, (Pharmarissano Arzneimittel GmbH, Bockenheim, Germany) $20 \mathrm{~g}$, sea salts (Sigma ${ }^{\circledR}$, S9883) 40 g per $\mathrm{L}$ of deionized $\mathrm{H}_{2} \mathrm{O}$. Culters grown on agar contained $20 \mathrm{~g} / \mathrm{L}$. All cultures were incubated at $25^{\circ} \mathrm{C}$ with $70 \%$ humidity with agitation at $220 \mathrm{rpm}$ or in static conditions (for solid cultures) unless stated otherwise. S. bicolor 293K04 was cultivated in twelve different liquid culture media: MMK2, YES, LSFM, XPMK (media recipes in [14]), Czapek $\left(\right.$ Difco $\left.^{\circledR}\right)$, MPY (malt, peptone and yeast medium) [15], BMS (described above), Malt 2\% [16], NPF2 [17], XLA [18], OP26-NLW [19] and SCY2 (undisclosed).

\subsubsection{Extraction of Cultures}

Strains were grown in 50-mL volume (in $250 \mathrm{~mL}$ Erlenmeyer flasks) or in 10-mL volume (EPA vials). For the 50-mL cultures, a $10 \mathrm{~mL}$ aliquot was transferred to an EPA vial solvent extraction prior to UV-LC-MS analysis. Equal volumes of acetone $(10 \mathrm{~mL})$ were added to each culture aliquot followed by agitation for $1 \mathrm{~h}$ at $220 \mathrm{rpm}$, after which $12 \mathrm{~mL}$ aliquots (out of the $20 \mathrm{~mL}$ total volume) of the extract supernatants were transferred to $15-\mathrm{mL}$ glass tubes (avoiding the transfer of fungal biomass). Afterwards, $600 \mu \mathrm{L}$ of DMSO were added to the samples (final concentration of $20 \%$ DMSO). Samples were concentrated to twice the initial culture volume ( $3 \mathrm{~mL}, 20 \% \mathrm{DMSO}$ ) under a $\mathrm{N}_{2}$ flow. Samples were transferred into microplate wells and centrifuged for $10 \mathrm{~min}$ at $2500 \mathrm{rpm}$ pellet cell debris.

\subsection{Ultraviolet-Liquid Chromatography-Mass Spectrometry (UV-LC-MS) Analysis}

\subsubsection{Low Resolution UV-LC-MS}

Two microliters of each extract were analysed by LC-MS unless stated otherwise. Samples were analysed on an Agilent (Santa Clara, CA, USA) 1100 single Quadrupole LC-MS, using a Zorbax SB-C8 column $(2.1 \times 30 \mathrm{~mm})$, maintained at $40^{\circ} \mathrm{C}$ and with a flow rate of $300 \mu \mathrm{L} / \mathrm{min}$. Solvent A consisted of $10 \%$ acetronitrile and $90 \%$ water with $0.01 \%$ trifluoroacetic acid and $1.3 \mathrm{mM}$ ammonium formate, 
while solvent B was $90 \%$ acetronitrile and $10 \%$ water with $0.01 \%$ trifluoroacetic acid and $1.3 \mathrm{mM}$ ammonium formate. The gradient started at $10 \%$ B and went to $100 \%$ B in $6 \mathrm{~min}$, kept at $100 \%$ B for $2 \mathrm{~min}$ and returned to $10 \% \mathrm{~B}$ for $2 \mathrm{~min}$ to initialize the system. Full diode array UV scans from 100 to $900 \mathrm{~nm}$ were collected in 4-nm steps at $0.25 \mathrm{~s} / \mathrm{scan}$. The eluting solvent was ionized using the standard Agilent $1100 \mathrm{ESI}$ source adjusted to a drying gas flow of $11 \mathrm{~L} / \mathrm{min}$ at $325^{\circ} \mathrm{C}$ and a nebulizer pressure of 40 psig. The capillary voltage was set to $3500 \mathrm{~V}$. Mass spectra were collected as full scans from $150 \mathrm{~m} / \mathrm{z}$ to $1500 \mathrm{~m} / \mathrm{z}$, with one scan every $0.77 \mathrm{~s}$, in both positive and negative modes.

\subsubsection{High Resolution UV-LC-MS}

Samples were analysed on an Agilent (Santa Clara, CA, USA) 1200 HPLC, using a Zorbax SB-C8 column $(2.1 \mathrm{~mm} \times 30 \mathrm{~mm})$, maintained at $40{ }^{\circ} \mathrm{C}$ and with a flow rate of $300 \mu \mathrm{L} / \mathrm{min}$. Solvent $\mathrm{A}$ consisted of $10 \%$ acetronitrile and $90 \%$ water with $0.01 \%$ trifluoroacetic acid and $1.3 \mathrm{mM}$ ammonium formate, while solvent B was $90 \%$ acetronitrile and $10 \%$ water with $0.01 \%$ trifluoroacetic acid and $1.3 \mathrm{mM}$ ammonium formate. The gradient started at $10 \% \mathrm{~B}$ and went to $100 \% \mathrm{~B}$ in $6 \mathrm{~min}$, kept at $100 \%$ B for $2 \mathrm{~min}$ and returned to $10 \%$ B for 2 min to initialize the system. Full diode array UV scans from 100 to $900 \mathrm{~nm}$ were collected in 4-nm steps at $0.25 \mathrm{~s} / \mathrm{scan}$.

Mass spectra were acquired on a Bruker maXis HR-QTOF mass spectrometer (Bruker Daltonics $\mathrm{GmbH}$, Bremen, Germany) coupled to the previously described LC system. The eluting solvent was ionized using the standard Maxis ESI source adjusted to a drying gas flow of $11 \mathrm{~L} / \mathrm{min}$ at $200{ }^{\circ} \mathrm{C}$ and a nebulizer pressure of $40 \mathrm{psig}$. The capillary voltage was set to $4000 \mathrm{~V}$. Mass spectra were collected from $150 \mathrm{~m} / z$ to $2000 \mathrm{~m} / z$ in positive mode.

\subsection{Methods for Time Course Measurements}

Culture time course measurements were based on five replicate measurements per time point from 50-mL cultivations (initially in $80 \times 250$-mL Erlenmeyer flasks). Every 2-3 d, during $37 \mathrm{~d}$ for, we recorded the $\mathrm{pH}$ (and $\mathrm{pH}$ measuring temperature) for each individual 50-mL culture and prepared an aliquot for organic extraction and UV-LC-MS analysis $(10 \mathrm{~mL}$ sample extracted and analyzed by UV-LC-MS, as described aboves). Dry weight biomass was determined from a 33-mL aliquot that was vacuum-filtered onto a pre-weighed paper filter $\left(\mathrm{VRW}^{\circledR} 150 \mathrm{~mm}\right.$ diam, qualitative paper, 413, particle retention $>5-13 \mu \mathrm{m}$ size). The paper filter with cells was dried at $60{ }^{\circ} \mathrm{C}$ for $24 \mathrm{~h}$. The dry weight was recorded and the biomass retained on the filter was calculated. All dry biomass values (recorded as $\mathrm{g} / 330 \mathrm{~mL}$ ), and raw data for $\mathrm{pH}$ and dry biomass are in Table S1.

\subsection{Radial Growth and Optimum Temperature Growth Measurements in Solid Medium}

Radial growth was measured in quintuplicate at six different temperatures during $69 \mathrm{~d}$ in solid BMS $(6 \times 5$ Petri plates, 90-mm diam); all culture plates were center-inoculated with a 5-mm mycelium agar plug of S. bicolor 293K04 previously grown in YME (yeast extract and malt extract) medium. The mycelial radial growth was measured in both the $\mathrm{Y}$ and $\mathrm{X}$ axis for each replicates (M1-M5) per temperature. Raw data, average growth and standard deviation values are available in the Table S3.

\subsection{Calibration Curves}

Pure endolide A and mariline A1/A2 (see Figure S1) were dissolved in methanol at initial concentrations of 500 and $250 \mathrm{mg} / \mathrm{L}$ respectively. Pure solutions (one volume of compound with one volume of methanol) were serially diluted until a final concentration of $0.24 \mathrm{mg} / \mathrm{L}$. Each dilution series was arrayed in a microplate and analyzed by UV-LC-MS $(1-\mu \mathrm{L}$ injection) as described above. We then assembled a graphic correlating the pure compounds concentration with the respective values of the pseudo molecular ion areas. A second specific calibration curve was assembled, ranging the pseudo molecular ion areas detected in the time course (see Figure S2). 


\section{Results}

\subsection{Production of Endolides by S. bicolor 293K04 during Short Incubation Times}

S. bicolor 293K04 was cultivated in 10-mL volumes in EPA vials using an array of 12 different liquid culture media. The media selection represented a broad variety of carbon and nitrogen source and varying concentrations of trace elements, and including as reference the liquid BMS medium previously used for 10-L of solid cultures. Cultures were grown in duplicate for 8 and $16 \mathrm{~d}$ and extracted with acetone to measure titres of secondary metabolites. Figure 2 shows the relative production in extracts for each of the 12 culture media at 8 and $16 \mathrm{~d}$. Endolide A was detected in all media tested at both times, whilst endolide B was only detected in Czapek medium at $8 \mathrm{~d}$ and further detected in five media at $16 \mathrm{~d}$. Production was generally low in liquid media. No major differences were found in the endolide production rates between the different media when compared with the BMS liquid culture, with the exception of Czapek medium. Endolides yields at $8 \mathrm{~d}$ were generally within the same range than the corresponding cultures extracted at 16 day. Finally, no polyketides were detected during any of these short incubation times.

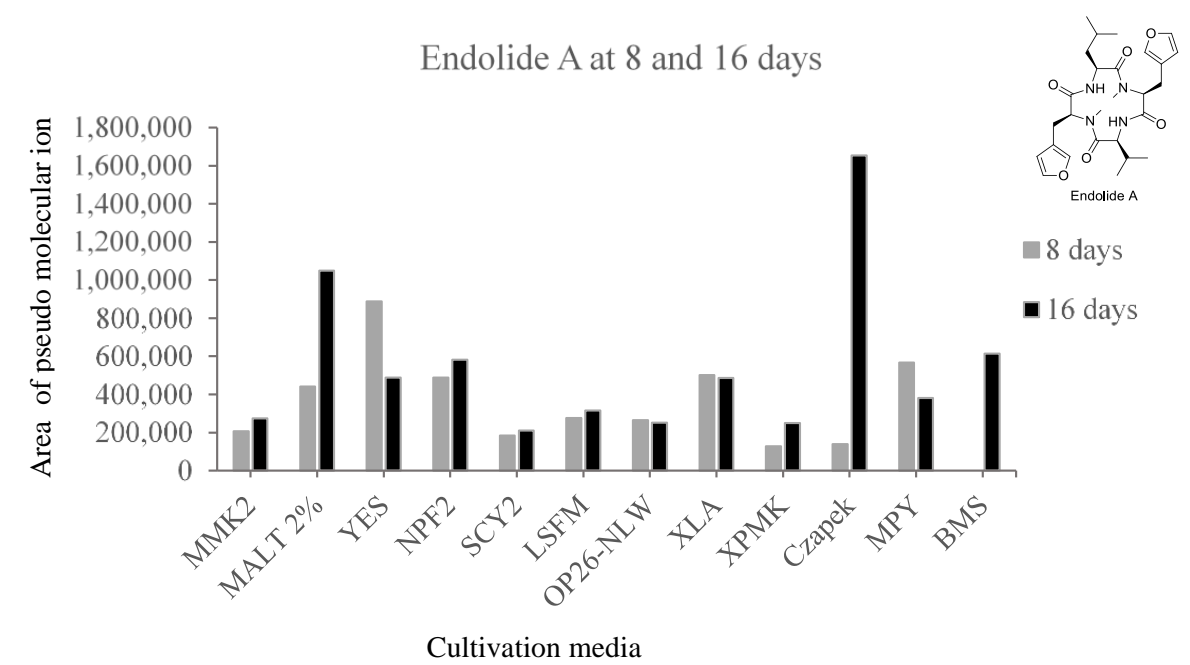

(a)

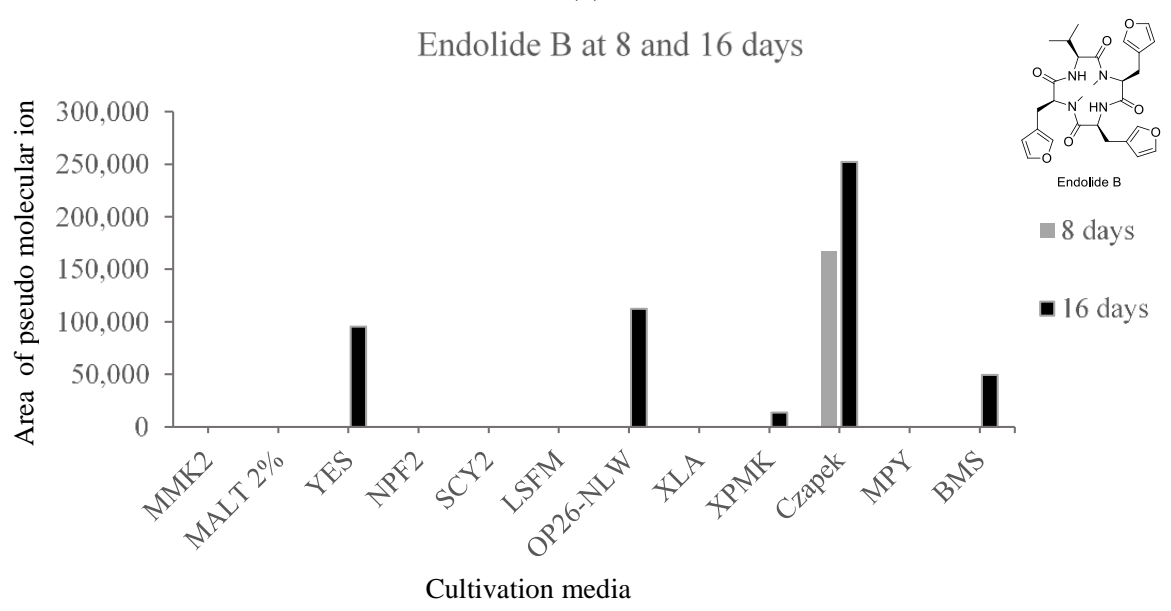

(b)

Figure 2. Secondary metabolite titers detected by UV-LC-MS in extracts of S. bicolor 293K04 from 8 (a) and $16 \mathrm{~d}$ (b) cultivations in 12 different liquid media grown in 10-mL volumes in EPA vials (see Table S2). $\mathrm{Y}$ values relates to the area of pseudo molecular ion corresponding to the integration of the extracted ion peaks, i.e., $515.3(\mathrm{M}+\mathrm{H})^{+}$for endolide $\mathrm{A}$ and $539.2(\mathrm{M}+\mathrm{H})^{+}$for endolide $\mathrm{B}$, with the retention times matching those of the pure endolides. 


\subsection{Time-Course Study for S. bicolor $293 K 04$}

A liquid formulation of BMS medium was selected to examine the production conditions of the endolides by S. bicolor 293K04. Cultures were grown in quintuplicate in flasks containing $50 \mathrm{~mL}$ of liquid medium. Liquid media permitted to follow the variability in biomass and $\mathrm{pH}$ with the goal of finding optimal conditions for endolide production. Given that we failed to detect the polyketides in any of the extracts of the same liquid nutrient media array, we decided to study the effect of cultivation parameters on the timing of the appearance and yields of these families of secondary metabolite in BMS medium liquid cultures in which the full spectrum of reported secondary metabolites had been previously isolated from solid cultures.

\subsubsection{Optimal Temperature for Growth of S. bicolor}

To determine the best growth temperature, we measured the radial growth of S. bicolor 293K04 with solid BMS at 18 to $30^{\circ} \mathrm{C}$ (Figure 3). After $69 \mathrm{~d}$ of incubation at the optimum temperature of $25^{\circ} \mathrm{C}$, the fungus had only covered, on average, $43 \%$ of the Petri dish.

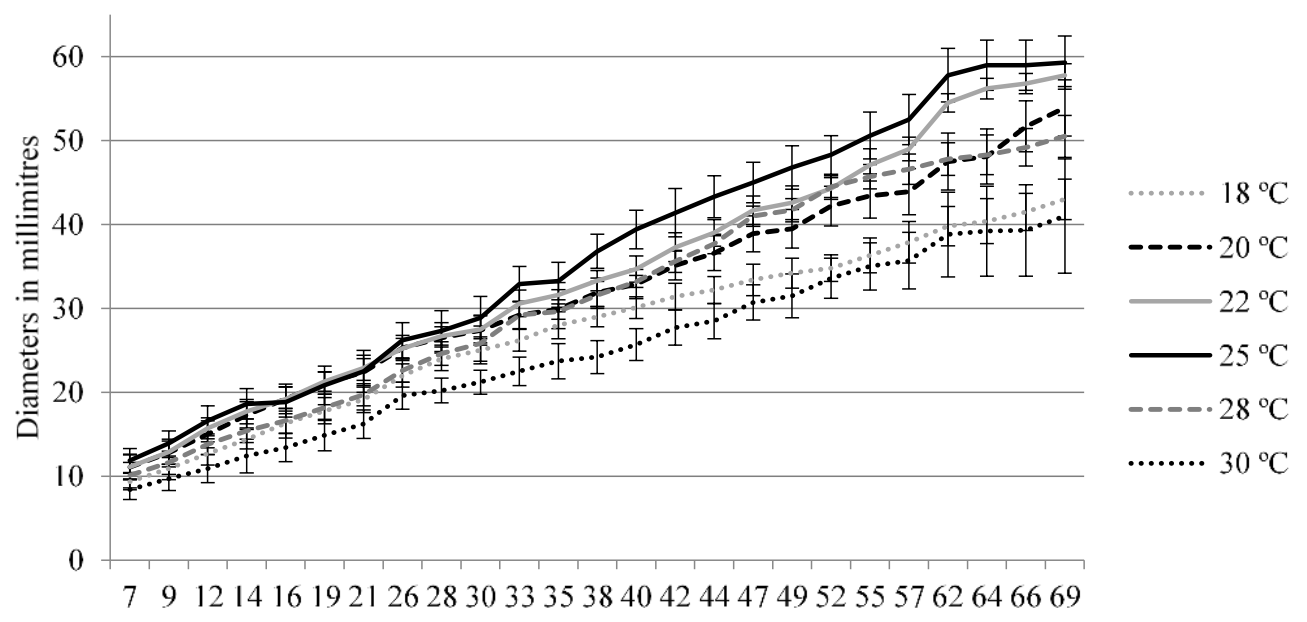

Cultivation time (days)

Figure 3. Radial growth curves of S. bicolor 293K04 in BMS medium at 6 temperatures. Radial growth was measured in quintuplicate at 2-3 days intervals after day 7 of incubation.

\subsection{2. $\mathrm{pH}$ and Biomass Variation during the Time Course}

In parallel to the secondary metabolite analysis, the $\mathrm{pH}$ and dry biomass were recorded during the time course study of S. bicolor $293 \mathrm{~K} 04$ fermented in liquid BMS for $37 \mathrm{~d}$ at $25^{\circ} \mathrm{C}$ (Figure 4).

Changes in $\mathrm{pH}$ over time behaved typically for fungal cultivations [20,21]. Extended incubation times resulted in medium acidification until 8-12 days, followed by basification at 23-26 days, with $\mathrm{pH}$ stabilization at 33-35 days. The $\mathrm{pH}$ time points at 8-12 days and 33-35 days roughly correlated with events in secondary metabolite production.

Biomass of S. bicolor 293K04 is low when grown in BMS medium. Biomass slowly increased throughout the time course, until $16 \mathrm{~d}$, after which it slowly decreased until day 37, although it did not affect secondary metabolite production (see below). 


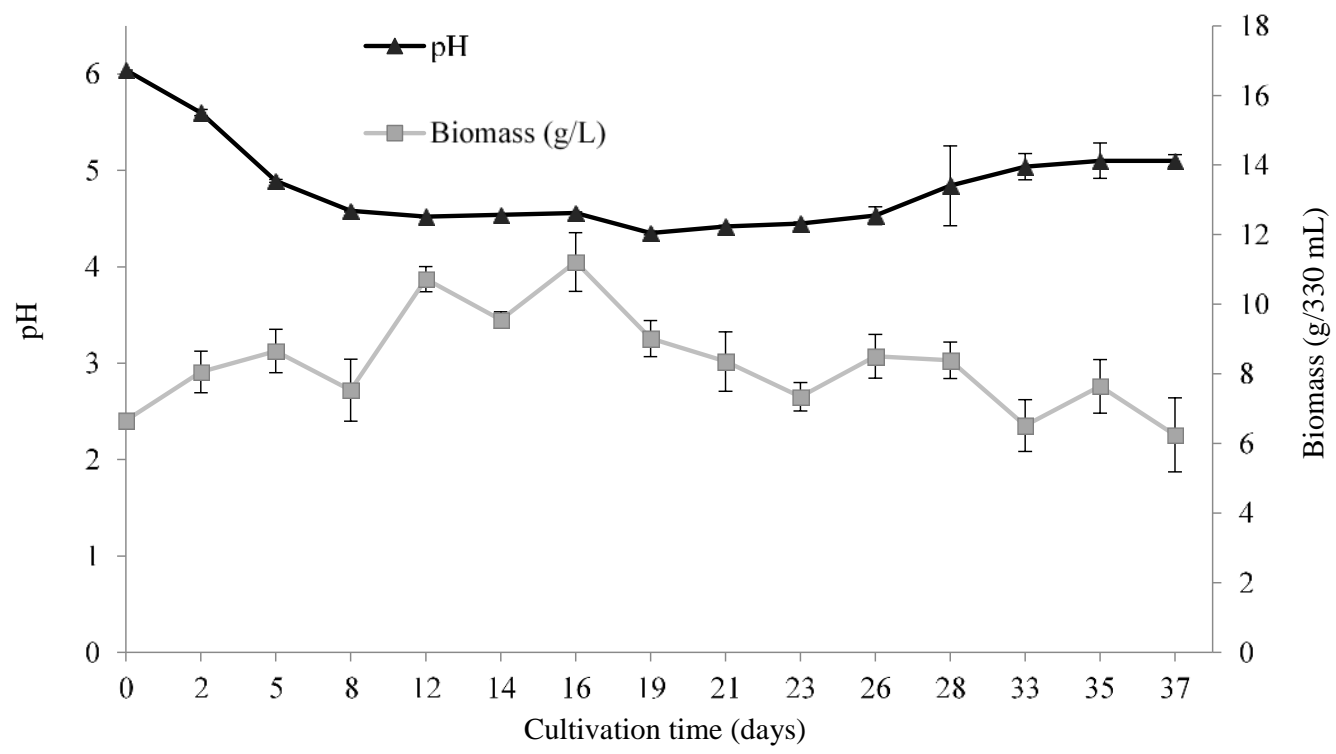

Figure 4. Evolution of $\mathrm{pH}$ and biomass $(\mathrm{g} / 330 \mathrm{~mL})$ of $S$. bicolor $293 \mathrm{~K} 04$ cultures in $50 \mathrm{~mL}$ of liquid medium for 37 days at $25^{\circ} \mathrm{C}$. Each point is the mean of 5 replicates.

\subsubsection{Endolides Production}

To determine the optimal production time for the endolides from S. bicolor 293K04 in liquid BMS medium, we followed their production during a period of 37 days by UV-LC-MS. Figure 5 shows that endolide A reached its maximum production after 28 days whereas the production of endolide $\mathrm{B}$ was only minimal after 8 days and did not increase with more time. Concentrations based on a calibration curve of pure endolide A indicated that the peak at 28 days was $<0.24 \mathrm{mg} / \mathrm{L}$ (see Figure S2). In prior experiments with 10-L solid BMS scale up cultivations, we isolated endolide A in ca. $5 \mathrm{mg} / \mathrm{L}$ yields and with endolide $B$ within the same range [3], i.e., values considerably higher than those achieved in liquid media. Endolide B production was estimated to be residual as shown in Figure 5. Time course values were measured with the area of the extracted pseudo molecular ions.

\subsubsection{Mariline A1/A2 Production}

Failure to detect the mariline polyketides in short incubations of nutrient array cultivations (Figure 2) and a late production of these compounds in 10-L solid cultures guided the analysis of their production at later times in the liquid cultures. The time course analysis showed the first detection of mariline A1/A2 at 35 days in only in one of the five replicate cultivations (Figure 6). However, polyketide production was detected in low amounts in all replicates at 37 days, results that are in agreement with the low yield registered in prior experiments in solid media after 40 days of growth, compared to the high polyketide yields measured from the 60-days solid cultures. The presence of mariline A1/A2 was confirmed by analyzing one replicate of a 37-d extract by HR-MS which identified the chemical formula of extracted ion peak.

A calibration curve with pure mariline A1/A2 was prepared for concentrations of $250 \mathrm{mg} / \mathrm{L}$ to $0.975 \mathrm{mg} / \mathrm{L}$. UV-LC-MS was sensitive to detect the pure compound at the lowest $0.5 \mathrm{mg} / \mathrm{L}$ and $0.24 \mathrm{mg} / \mathrm{L}$ concentrations. Our interpretation is that mariline A1/A2 seems to have a lower capacity for ionization by ESI (see Figure S2). Comparing the titers of mariline A1/A2 at 37 days, i.e., the area of the pseudo molecular ion, with the calibration curve of the pure compound, we could only assume that the residual yield of mariline A1/A2 at 37 days was $<0.975 \mathrm{mg} / \mathrm{L}$. Because of the low detected values and early production of the compound, the results (Figure 6) were presented as the area of the pseudo molecular ion. No other polyketide was detected during the time course, hence, we can assume mariline A1/A2 was the end product of polyketide biosynthesis. 


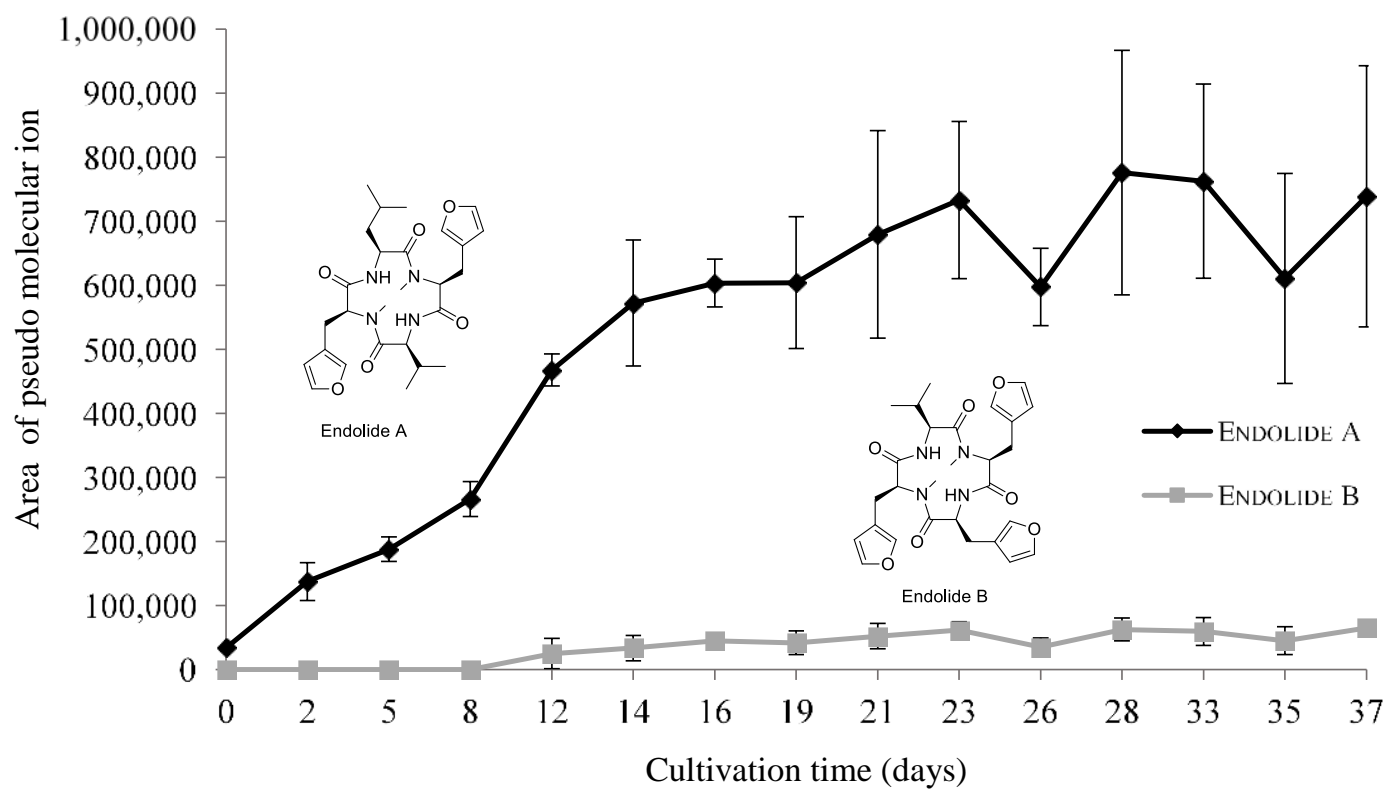

Figure 5. Endolide A and B production by S. bicolor 293K04 cultivated in BMS liquid medium. Each time point corresponds to five $50 \mathrm{~mL}$ cultures. Compounds were detected by LC-MS after whole broth acetone extraction at 2-3 days intervals during 37 days. Extracts were concentrated two-fold from the initial culture volume. Production is represented as the area of molecular ion corresponding to the integration of the extracted ion peaks, $515.3(\mathrm{M}+\mathrm{H})^{+}$for endolide A and $539.2(\mathrm{M}+\mathrm{H})^{+}$for endolide B. Secondary metabolite yields were measured as the extracted ion peak area, (Raw UV-LC-MS data in Supporting Information).

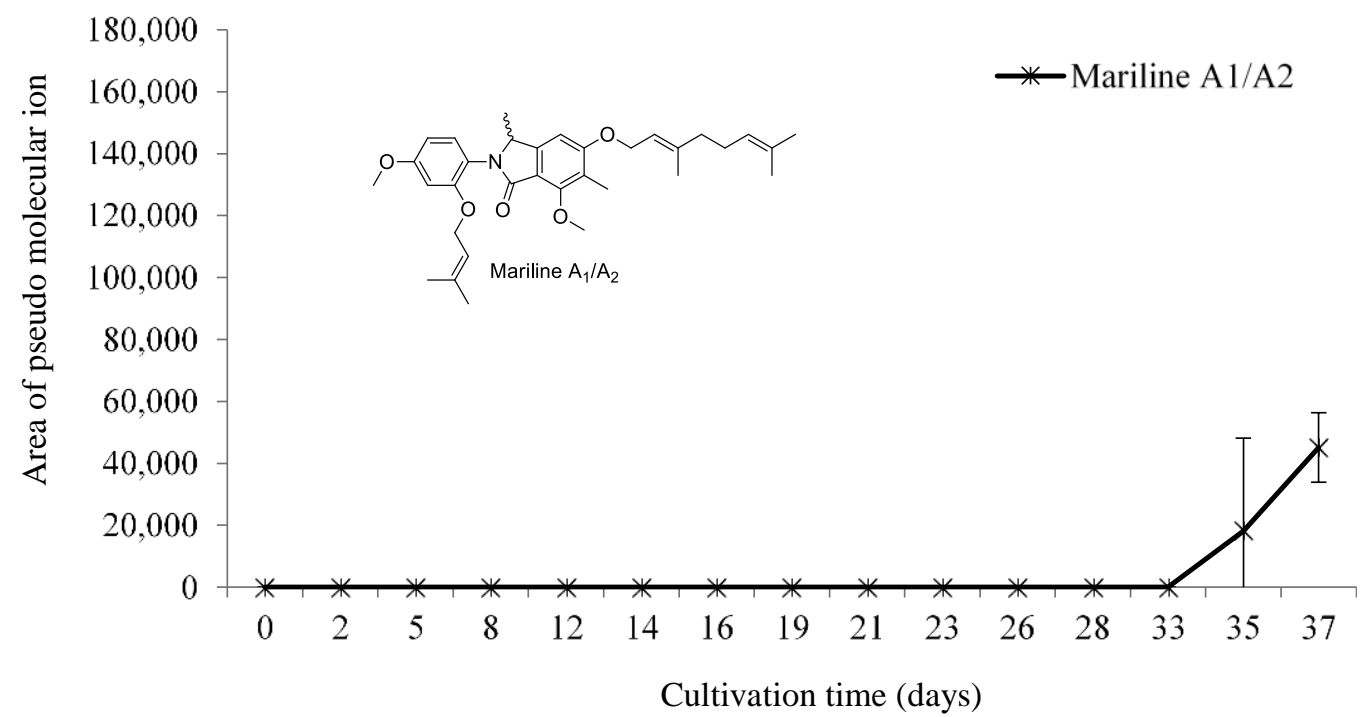

Figure 6. Time course analysis of mariline A1/A2 production by S. bicolor 293K04 grown in BMS. Each time point corresponds to the analysis of five $50-\mathrm{mL}$ cultures. Compounds were detected by LC-MS after whole broth acetone extracts collected at 2-3 days intervals during 37 days. Cultivation extracts were concentrated twice the initial culture volume. $Y$ values are the areas of pseudo molecular ion corresponding to the integration of the extracted ion peaks, $534.3(\mathrm{M}+\mathrm{H})^{+}$for mariline A1/A2. UV-LC-MS raw data in Supporting Information. 


\section{Discussion}

The time course study for the optimization of the endolides yields in liquid cultures evidenced a typical production curve for fungal secondary metabolites. The initial production of both endolides production at $12 \mathrm{~d}$ seemed to be correlated with the stabilization of the culture $\mathrm{pH}$ at 4.5.

Solid cultures seemed to be the preferred cultivation method for isolating high yields of the peptidic endolides, with around a 30-fold lower yield of endolide A in liquid BMS when compared with solid BMS medium, and an estimated residual production of endolide B in liquid BMS cultures, which may be due to a different physiology of S. bicolor 293K04 in liquid versus solid cultures. S. bicolor 293K04 grows poorly in liquid BMS medium. Although it did not affect the secondary metabolite production, the physiology of the fungus in submerged cultures was suboptimal for these secondary metabolites when comparing the production yields observed in solid cultures. To address the problem of the limited amounts of endolides production for in vivo bioassays, new nutrient combinations will need to be tested in liquid cultures to identify a high-yield producing media that could be used to scale up cultures.

The time course evidenced the late stage mariline A1/A2 production by S. bicolor 293K04, further confirming the previous results where new polyketides were identified using an experimental 60-d solid culture which yielded higher titers. We have correlated the plateau of the late stationary phase $\mathrm{pH}$ increase with the beginning of polyketide production. Activation of gene clusters encoding for secondary metabolites at such late cultivations times has been rarely reported in fungi, as such time-extended cultures are not frequently considered. A previous report has shown that extended cultivation of Aspergillus strain CMB-M81F over 120 and 160-d periods enhanced secondary metabolite expression whereas shorter cultivation times showed no meaningful secondary metabolite profile [22]. Two new secondary metabolites were also reported from these cultures, shornepine A and the diketopiperazine $15 \mathrm{~b}-\beta$-methoxy-5- $N$-acetyladreemin [23]. In control cultures, shornephine A, first appeared at $70 \mathrm{~d}$, with maximum production at $160 \mathrm{~d}$. Although a new natural product was reported from this Aspergillus strain CMB-M81F, the effects and culture conditions for almost 6 months, one needs to consider if such long fungal cultivations are worthwhile, not only because such extremely long cultivation times are not sustainable in terms of convenience for bioactive natural products research, but also from the microbiological aspect because long-term effects on cell viability are generally lacking. Also, reproducibility of secondary metabolite production may be difficult to attain in such long-term cultures, especially when a large numbers of strains are grown in such a variable situation. Interestingly, an extended time course study for 45 days induced lignolytic enzyme production of Trametes versicolor at the late stages of cultivation [21], further supporting that cells are viable and physiologically active at extended cultivation times.

It is generally accepted that long-term liquid fungal cultivations where cultures are maintained well beyond the stationary phase and into the autolytic stage would present an unfavourable environment because of carbon or nitrogen starvation, elevated $\mathrm{pH}$ due to the uptake of excreted organic acids by the mycelium, scavenging and translocation of nutrients from polymers within the mycelium, accumulation of self-toxic metabolites, and degeneration or autolysis of mycelium. These unfavourable conditions for fungal growth are generally thought to adversely affect secondary metabolite expression [24]. Therefore, fungal strains are frequently cultivated for relatively short growth periods of one to three weeks which generally coincides with the time needed for robustly growing fungi to reach late exponential or stationary phase in liquid cultures. An alternative approach to determining harvest time for screening cultivations has been to monitor glucose concentrations in the culture broth with a glucose assay kit, and extract the cultures when the glucose is exhausted $[25,26]$. Sugar concentration was measured in the herein report, as we saw that maltose in the whole broth extracts was not detectable in the extracts by ESI-LC-MS.

The above results indicated that long-term growth of fungal cultures may favour production and detection of secondary metabolites, possibly produced in response to culture-induced stresses that are not generally observed in short-grown cultures. In light of the current interest in finding 
alternative ways to stimulate poorly expressed biosynthetic pathways in fungi, the idea of using extended cultivation times as a means to stimulating expression of cryptic metabolites merits further investigation. Although endolides were produced, despite the low yield, as expected in fungal metabolism, the onset of the polyketide production in S. bicolor 293K04 did not follow the canonical pattern of secondary metabolism where maximum yields coincide with the onset of the stationary phase of a batch culture. When we integrate all of the above results into a single temporal sequence of growth and onset of different metabolites, we envisage a two-phase pattern for secondary metabolism in S. bicolor 293K04 with a first week dominated by the endolide gene clusters expression, and the second period dominated by polyketide production with a decay in peptide titers as indicated by the low quantities of endolides isolated from the 60-d 10-L scaled up solid cultivation.

Our knowledge about the longevity of fungal growth have been skewed by laboratory experiments where strains are grown in high nutrient conditions in confined axenic environments, and where the need to be expeditious and efficient in providing microbial compounds for study of their bioactive properties pressures investigators to conveniently standardize shorter laboratory growth cycles. The above results and analysis raise some interesting questions about the potential to explore the autolytic phase of a culture for newly formed secondary metabolites. Extending the study of the cultivation of S. bicolor $293 \mathrm{~K} 04$ beyond a 60-d time course development could further elucidate the polyketides production patterns and optimal peak production. Such exploration should be accompanied by a full study of parameters related to the viability of the fungal culture in such conditions. It is noteworthy that several other polyketides that were isolated from the 60-d 10-L solid culture extracts remain to be characterized due to insufficient yields for 2D-NMR spectroscopic characterization. These compounds appear to be new due to the presence of the characteristic methyl group in the starter unit found in these polyketides, a feature previously unseen in reported phthalimidine/phthalide core structures.

Supplementary Materials: The following are available online at www.mdpi.com/2311-5637/3/3/45/s1, Figure S1: UV-LC-MS detection chromatograms for the pure chemical family representatives of S. bicolor 293K04 isolated secondary metabolites, namely endolides A-B and mariline A1/A2, Table S1: Data from the time course study, Figure S2: Calibration curves, Table S2: UV-LC-MS secondary metabolite detection after 8 and 16 days cultivation of the S. bicolor 293K04 in a nutrient array of 12 culture media, Table S3: Radial growth rates in the 69-day solid BMS medium time course of S. bicolor $293 \mathrm{~K} 04$.

Acknowledgments: We would like to thank funding from Fundação para a Ciência e Tecnologia, FCT, Portugal (fellowship SFRH/BPD/77720/2011). This research was financially supported by Fundación MEDINA.

Author Contributions: Celso Almeida conducted the overall experiments, analyzed the data and wrote the paper; Gerald Bills conceived and designed the experiments, wrote, and substantively revised the paper; Víctor González-Menéndez grew the fungi and co-designed the experiments; Jesús Martin conducted the acquisition and interpretation of UVLC-MS data, José R. Tormo conducted the acquisition of organic extracts and data interpretation; Olga Genilloud co-developed the experimental design and substantively revised the paper.

Conflicts of Interest: The authors declare no conflict of interest.

\section{References}

1. Brakhage, A.A. Regulation of fungal secondary metabolism. Nat. Rev. Microbiol. 2013, 11, 21-32. [CrossRef] [PubMed]

2. Andersen, M.R.; Nielsen, J.B.; Klitgaard, A.; Petersen, L.M.; Zachariasen, M.; Hansen, T.J.; Blicher, L.H.; Gotfredsen, C.H.; Larsen, T.O.; Nielsen, K.F.; et al. Accurate prediction of secondary metabolite gene clusters in filamentous fungi. Proc. Natl. Acad. Sci. USA 2013, 110, E99-E107. [CrossRef] [PubMed]

3. Almeida, C.; El Maddah, F.; Kehraus, S.; Schnakenburg, G.; Konig, G.M. Endolides A and B, vasopressin and serotonin-receptor interacting n-methylated peptides from the sponge-derived fungus Stachylidium sp. Org. Lett. 2016, 18, 528-531. [CrossRef] [PubMed]

4. Potgieter, M.; Steyn, P.S.; van Heerden, F.R.; van Rooyen, P.H.; Wessels, P.L. Conformational analysis of the cyclic peptide rhizonin a in solution and crystalline state. Tetrahedron 1989, 45, 2337-2350. [CrossRef] 
5. Steyn, P.; Tuinman, A.A.; van Heerden, F.R.; van Rooyen, P.H.; Wessels, P.L.; Rabie, C.J. The isolation, structure, and absolute configuration of the mycotoxin, rhizonin a, a novel cyclic heptapeptide containing $\mathrm{N}$-methyl-3-(3-furyl)alanine, produced by rhizopus microsporus. J. Chem. Soc. Chem. Commun. 1983, 47-49. [CrossRef]

6. Partida-Martinez, L.P.; de Looss, C.F.; Ishida, K.; Ishida, M.; Roth, M.; Buder, K.; Hertweck, C. Rhizonin, the first mycotoxin isolated from the zygomycota, is not a fungal metabolite but is produced by bacterial endosymbionts. Appl. Environ. Microbiol. 2007, 73, 793-797. [CrossRef] [PubMed]

7. Xiang, W.S.; Wang, J.D.; Wang, X.J.; Zhang, J. Bingchamides A and B, two novel cyclic pentapeptides from the streptomyces bingchenggensis: Fermentation, isolation, structure elucidation and biological properties. J. Antibiot. 2009, 62, 501-505. [CrossRef] [PubMed]

8. Almeida, C.; Eguereva, E.; Kehraus, S.; Konig, G.M. Unprecedented polyketides from a marine sponge-associated stachylidium sp. J. Nat. Prod. 2013, 76, 322-326. [CrossRef] [PubMed]

9. Almeida, C.; Hemberger, Y.; Schmitt, S.M.; Bouhired, S.; Natesan, L.; Kehraus, S.; Dimas, K.; Gutschow, M.; Bringmann, G.; Konig, G.M. Marilines A-C: Novel phthalimidines from the sponge-derived fungus Stachylidium sp. Chemistry 2012, 18, 8827-8834. [CrossRef] [PubMed]

10. Almeida, C.; Kehraus, S.; Prudencio, M.; Konig, G.M. Marilones a-c, phthalides from the sponge-derived fungus Stachylidium sp. Beilstein J. Org. Chem. 2011, 7, 1636-1642. [CrossRef] [PubMed]

11. Almeida, C.; Part, N.; Bouhired, S.; Kehraus, S.; Konig, G.M. Stachylines A-D from the sponge-derived fungus Stachylidium sp. J. Nat. Prod. 2011, 74, 21-25. [CrossRef] [PubMed]

12. Calvo, A.M.; Wilson, R.A.; Bok, J.W.; Keller, N.P. Relationship between secondary metabolism and fungal development. Microbiol. Mol. Biol. Rev. 2002, 66, 447-459. [CrossRef] [PubMed]

13. Hahn-Hägerdal, B.; Karhumaa, K.; Larsson, C.U.; Gorwa-Grauslund, M.; Görgens, J.; van Zyl, W.H. Role of cultivation media in the development of yeast strains for large scale industrial use. Microb. Cell Fact. 2005, 4, 31. [CrossRef] [PubMed]

14. González-Menéndez, V.; Asensio, F.; Moreno, C.; de Pedro, N.; Monteiro, M.C.; de la Cruz, M.; Vicente, F.; Bills, G.F.; Reyes, F.; Genilloud, O.; et al. Assessing the effects of adsorptive polymeric resin additions on fungal secondary metabolite chemical diversity. Mycology 2014, 5, 179-191. [CrossRef] [PubMed]

15. Harms, H.; Orlikova, B.; Ji, S.; Nesaei-Mosaferan, D.; Konig, G.M.; Diederich, M. Epipolythiodiketopiperazines from the marine derived fungus dichotomomyces cejpii with nf-kappab inhibitory potential. Mar. Drugs 2015, 13, 4949-4966. [CrossRef] [PubMed]

16. Serrano, R.; González-Menéndez, V.; Rodríguez, L.; Martín, J.; Tormo, J.R.; Genilloud, O. Co-culturing of fungal strains against botrytis cinerea as a model for the induction of chemical diversity and therapeutic agents. Front. Microbiol. 2017, 8, 649. [CrossRef] [PubMed]

17. De la Cruz, M.; Martin, J.; Gonzalez-Menendez, V.; Perez-Victoria, I.; Moreno, C.; Tormo, J.R.; El Aouad, N.; Guarro, J.; Vicente, F.; Reyes, F.; et al. Chemical and physical modulation of antibiotic activity in emericella species. Chem. Biodivers. 2012, 9, 1095-1113. [CrossRef] [PubMed]

18. El Aouad, N.; Perez-Moreno, G.; Sanchez, P.; Cantizani, J.; Ortiz-Lopez, F.J.; Martin, J.; Gonzalez-Menendez, V.; Ruiz-Perez, L.M.; Gonzalez-Pacanowska, D.; Vicente, F.; et al. Lasionectrin, a naphthopyrone from a lasionectria sp. J. Nat. Prod. 2012, 75, 1228-1230. [CrossRef] [PubMed]

19. Basilio, A.; Justice, M.; Harris, G.; Bills, G.; Collado, J.; de la Cruz, M.; Diez, M.T.; Hernandez, P.; Liberator, P.; Nielsen kahn, J.; et al. The discovery of moriniafungin, a novel sordarin derivative produced by morinia pestalozzioides. Bioorg. Med. Chem. 2006, 14, 560-566. [CrossRef] [PubMed]

20. Moreira Neto, S.L.; Matheus, D.R.; Machado, K.M.G. Influence of ph on the growth, laccase activity and rbbr decolorization by tropical basidiomycetes. Braz. Arch. Biol. Technol. 2009, 52, 1075-1082. [CrossRef]

21. Yamanaka, R.; Soares, C.F.; Matheus, D.R.; Machado, K.M.G. Lignolytic enzymes produced by trametes villosa ccb176 under different culture conditions. Braz. J. Microbiol. 2008, 39, 78-84. [CrossRef] [PubMed]

22. Khalil, Z.G.; Kalansuriya, P.; Capon, R.J. Lipopolysaccharide (lps) stimulation of fungal secondary metabolism. Mycology 2014, 5, 168-178. [CrossRef] [PubMed]

23. Khalil, Z.G.; Huang, X.C.; Raju, R.; Piggott, A.M.; Capon, R.J. Shornephine a: Structure, chemical stability, and p-glycoprotein inhibitory properties of a rare diketomorpholine from an australian marine-derived aspergillus sp. J. Org. Chem. 2014, 79, 8700-8705. [CrossRef] [PubMed]

24. Griffin, D.H. Fungal Physiology, 2nd ed.; John Wiley \& Sons, Ltd.: New York, NY, USA, 1995. 
25. Kettering, M.; Sterner, O.; Anke, T. Antibiotics in the chemical communication of fungi. Z. Naturforsch. C J. Biosci. 2004, 59, 816-823. [CrossRef]

26. Weber, D.; Sterner, O.; Anke, T. Mollisianitrile, a new antibiotic from mollisia sp. A59-96. Z. Naturforsch. C J. Biosci. 2007, 62, 567-570. [CrossRef]

(c) (C) 2017 by the authors. Licensee MDPI, Basel, Switzerland. This article is an open access article distributed under the terms and conditions of the Creative Commons Attribution (CC BY) license (http:/ / creativecommons.org/licenses/by/4.0/). 